See discussions, stats, and author profiles for this publication at: https://www.researchgate.net/publication/304702855

\title{
Development of a User Interface for the Enrichment of Situational Awareness in Emergency Management Systems
}

Chapter · January 2016
DOI: 10.1007/978-3-319-41929-9_1

\section{CITATIONS}

2

5 authors, including:

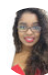

Natália Oliveira

University of São Paulo

11 PUBLICATIONS 12 CITATIONS

SEE PROFILE

Jessica Oliveira De Souza

São Paulo State University

16 PUblications 25 Citations

SEE PROFILE
Fabio Rodrigues Jorge

University of São Paulo

6 PUBLICATIONS 14 CITATIONS

SEE PROFILE

Valdir Pereira Junior

Centro Universitário Eurípides de Marília

12 PUBLICATIONS 17 CITATIONS

SEE PROFILE

Some of the authors of this publication are also working on these related projects: 


\title{
Development of a User Interface for the Enrichment of Situational Awareness in Emergency Management Systems
}

\author{
Natália Oliveira ${ }^{1}$, Fábio Rodrigues Jorge ${ }^{1}$, Jéssica Souza ${ }^{2}$, Valdir Júnior ${ }^{1}$, Leonardo \\ Botega $^{1}$ \\ ${ }^{1}$ Marília Eurípides University, Marília, São Paulo, Brazil \\ ${ }^{2}$ São Paulo State University “Júlio Mesquita Filho”, Marília, São Paulo, Brazil \\ \{nataliaoliveira, binho, valdir.junior, botega\}@univem.edu.br, \\ osz.jessica@marilia.unesp.br, regina@dc.ufscar.br
}

\begin{abstract}
The individual's perception and understanding obtainment on events that transform an environment and their real critical contexts, is named Situational Awareness (SA). This process assists emergency management situation, allowing experts on SA acquisition and maintenance process, and provide subsidies for an assertive decision making. Specialized SA User Interfaces (UI) may facilitate the knowledge acquisition, allowing the perception and dynamic comprehension. The UIs design of SA-oriented systems in critical scenarios, as emergency management is a challenging issue, considering the presence of multiple heterogeneous data source, the information about situation that's in constant transformation and quality limitations associated every new inference. Known approaches seek to present specifically solutions to their application domain and are inevitably limited as the described needs in this paper. This paper presents the UI development to promote the visualization and situational information evolution follow-up, information quality aware, driven to a case of study in emergency management, which includes new challenges like the data presentation from human intelligence.
\end{abstract}

Keywords: Situational Awareness · User Interface - Emergency Systems Assessment.

\section{$1 \quad$ Introduction}

Endsley [1] defines Situational Awareness (SA) as a process based on perception and comprehension development about what is happening in the environment inside of a volume and space of time, and the projection of its status in the near future.

\footnotetext{
${ }^{*}$ Please note that the LNCS Editorial assumes that all authors have used the western naming convention, with given names preceding surnames (first name then last name). This determines the structure of the names in the running heads and the author index. No academic titles or descriptions of academic positions should be included in the addresses. The affiliations should consist of the author's institution, town, and country.
} 
The decision making systems, as emergency management systems, are recognized by experts as base provider to support the situational perception.

In critical scenarios, such emergency calls domain, a SA poor may harm the situation comprehension, compromising the allocation of resources, the heritage and the environment preservation. Furthermore, the SA process improvement may support operational process, assisting on the strategic actions planning, improving decisions quality and allowing better scenario comprehension.

Define a graphic representation process for critical situation management domain, is a challenge issue on Situation Assessment community, given the dynamics and complexity of information representation [2]. Moreover, provides data to experts about information's quality, to acquire a better comprehension about what is happening on the environment, are factors that assists the decision making process [3].

The data quality in assessment of emergency situations is also one of the critical factors in the decision making process [4], whereas situation awareness process may be impaired, have reduced effectiveness when imperfections information is input into the process, contributing negatively with the formation the operator mental model to respond to emergency calls.

In this paper is introduced the UI development dedicated to support of emergency situation data and information representation and management, quality aware, produced by emergency management system. A case of study addressing the analysis an emergency management domain is characterized, in order to illustrate as the use of UI can contribute to the situation assessment process.

\section{Interfaces for Emergency Management}

This section presents the state-of-the-art for SA-oriented interfaces. Existing solutions typically aim to empower the operator and the system by intensifying their relationship with information to build a more feasible representation of situations.

Yu et al. [5] presented a new visualization context through the UI, which has an interpretation engine for the operator's needs, defining which information must be presented. For the improvement of the operator's comprehension, a mechanism of fuzzy control was proposed to perform a diffuse search based on specific keywords of the application domain by operators' interactions.

Feng et al. [6] developed a decision support system that incorporates shared SAW among agents that extract relevant information about entities and represent them to the operator. These agents have the following set of goals and strategies for each SAW level: missions, plans, actions and physical attributes. Then, they are responsible for generating recommendations about the scenario. The UI deals with the spatialtemporal aspects of the evolution of missions.

Besides being efficient solutions for the specific application domains, such solutions are limited regarding the management of information being propagated throughout the cycle of situation assessment. Our approach innovates on promoting a full control of the information that is produced on each phase, using uncertainty representation and refinement methods as a resource to control the knowledge that is created, represented and used to assess situations. 
The next chapter presents the interface development applied the Endsley [9][10] guidelines, as well each item choices justifications.

\section{The development of the user interface for situational assessment systems}

Situation Assessment has as the primary responsibility produce subsidies to assists the human operator's SA and consequently generate knowledge to the decision making process. A situation assessment complete process in complex scenarios it is intended to acquire, process and present parts of information, can contribute with situation understanding.

The process starts on Data Acquisition module, where the transcript of audio reported to the São Paulo State Police (PMESP) via emergency calls, and the social network posts acquisition is made. At this, a grammatical analysis to identify relevant objects, attributes and properties in sentences is performing, process called Natural Language Processing (NLP). A set of objects, attributes and properties called Situation, is produced and subject to the following module to the data and information quality assessment.

Data and Information Quality Assessment module determines a sequence of activities to quality data presents. Herein, situation is submitted to following dimensions' analysis: describing data or attributes completeness; currency, helps determine the information "age"; and uncertainty, a generalization of others dimensions in a single quality measure (set of data). Thus, each object has its completeness score, and in case of failure, time and uncertainty scores are applied [7].

On Situation Knowledge Representation Module, the goal is present semantic information, owing to flexibility present, object's relations in each class, called Object Propriety.

The Data and Information Fusion Module, performs search among the synergic information between present classes on ontology, that may hold object, attributes, proprieties and quality scores information's which have matching. As result, submitted information to fusion process are analyzed to find synergies, hierarchically, resulting new situational information [8].

Ultimately, SA-oriented User Interface Module, aims determines a sequence of activities to assists critical situations management process. This work, acts in this situational assessment stage. The UI, aims allow experts to SA acquire, maintain, and recover. This stage should present acquire, process, if necessary fused and present situations significantly

The next chapter, the UI development is present and a case of study applied. This, refers to an urban robbery situation, related to PMESP's central monitoring complains, and the information inclusion on critical situations management process.

The case of study, guides this work development, describe an urban robbery complains input and process occurred in São Paulo/Brazil. The complains arrives to PMESP central, across emergency calls or social network posts. Each new complains, the system operator receives situational graphic representation actualization starting objects which comprise interest environment (the victim, the criminal, the stolen object, and event spot) and complainants present attributes and identified by the tool. 
For the UI development lifting requirements, guidelines to SA design, introduced by Endsley [9] [10] were adopted. Thus, in this section are presented the development guidelines for each UI component and state of the arte design choices. Highlights up advantages and disadvantages each design choice for urban critical scenario.

In order to starts interface with information, our case of study will be addressing the following situation: "To stop the car on Domingos Setti avenue with Luis Vives street, at 2 PM, Luciano was surprised for an armed individual by an unidentified object. He was forced to out of the car with his hands up, but due to his age and walking difficulties, Luciano took some time to perform the robbery order, and in this way was assaulted hard head. Injured, Luciano get out of the car and tall individual, using light blue jeans and yellow t-shirt, run away with Luciano's black Porshe direction west of the city to Klabin subway. To be a little traffic local, they weren't registered many reports related to this incident."

\subsection{Organize information according to the goals}

The goal is analysis and evaluate situations from emergency management situations domain, to assists experts SA acquire using collated and processed information starting assessment systems.

For that, the information presentation was structured around goals to build a faithful situation presentation and follow the evolution, thus, it's necessary that UI do not totally goal-driven oriented, aiming SA contribution. Hence, the interface was divided into three different but inter-connected views. Fig. 1 presents the UI completed visualization for the acquisition of SA.

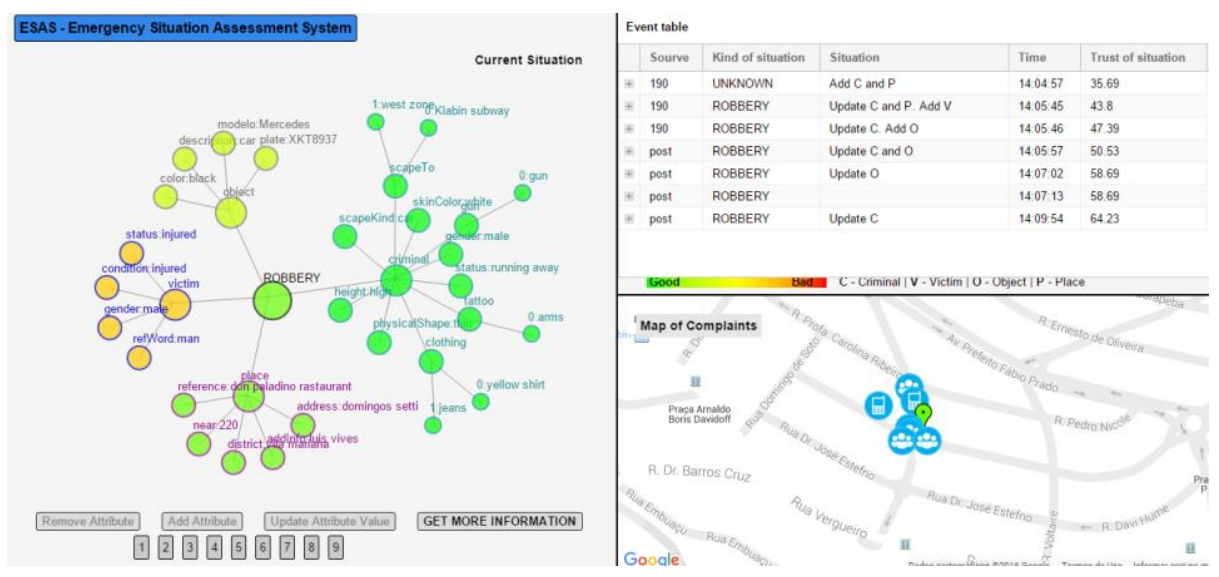

Fig. 1 - UI presentation to emergency management.

The first view (top right) in the UI is an object's table for incoming events, containing: information source, type of situation, objects found by acquisition and fusion, added time in-formation and the information quality (0 to 100) assessed. In this table was specificity the transformations, like: object data actualization.

Fig. 2, a report that occurs at 2:04 PM is exhibited: "just happened a criminal here in domingos setti a driver was threatened and ordered he come out of vehicle with 
nothing the robbery run away toward the Klabin subway" this are identified the information source, being an emergency call, type of situation that so far it is unknown, and could be add objects types: criminal and event spot, because ate the report this information was provided, feeding the situation.

Event table

\begin{tabular}{|c|c|c|c|c|c|}
\hline & Sourve & Kind of situation & Situation & Time & Trust of situation \\
\hline \multirow[t]{2}{*}{-} & 190 & UNKNOWN & Add $C$ and $P$ & $14: 04: 57$ & 35.69 \\
\hline & \multicolumn{5}{|c|}{$\begin{array}{l}\text { More information: just happened a crime here on Domingos Setti a driver was threatened and ordered } \\
\text { him out of the vehicle without taking anything bandit fled toward the Klabin subway }\end{array}$} \\
\hline+ & 190 & ROBBERY & Update C and P. Add V & $14: 05: 45$ & 43.8 \\
\hline+ & 190 & ROBBERY & Update C. Add O & $14: 05: 46$ & 47.39 \\
\hline+ & post & ROBBERY & Update $\mathrm{C}$ and $\mathrm{O}$ & $14: 05: 57$ & 50.53 \\
\hline+ & post & ROBBERY & Update O & $14: 07: 02$ & 58.69 \\
\hline+ & post & ROBBERY & & $14: 07: 13$ & 58.69 \\
\hline
\end{tabular}

Fig. 2 - First view, presentation of object's table for incoming events.

The second view (bottom right) is a map-based, with visualizations as overlays geo-located according to the location of the acquired data. The use of georeferenced map overlays, is used in emergency management operations, because there is localization dependence to determine the attendance of the event, as shown in Fig. 3.

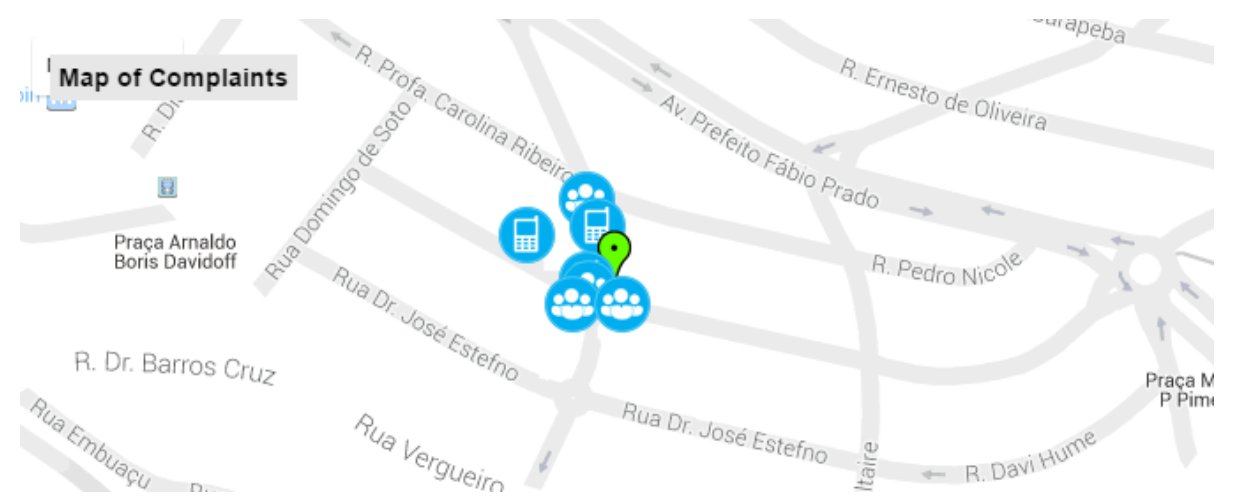

Fig. 3 - Second view, map presentation.

Based on case of study, ate the map are present the event spot approximated localizations identified in reports. The red marker presents the average found localization, and the blue glyphs presents de made reports localizations, differentiated by types, like: social network or emergency calls. 
The third view (left) comprises for a relational graph, justified by the need of information hierarchical knowledge. Situation is present by the central node, with relations between objects and attributes.

The node size presents your importance; the biggest node presents the situation. The next level presents the set of objects (entities) identified on inference (ex: local). The smaller node leaves present the objects attributes (ex: reference points, descriptions, status), As show in Fig.4.

\section{ESAS - Emergency Situation Assessment System}

Current Situation

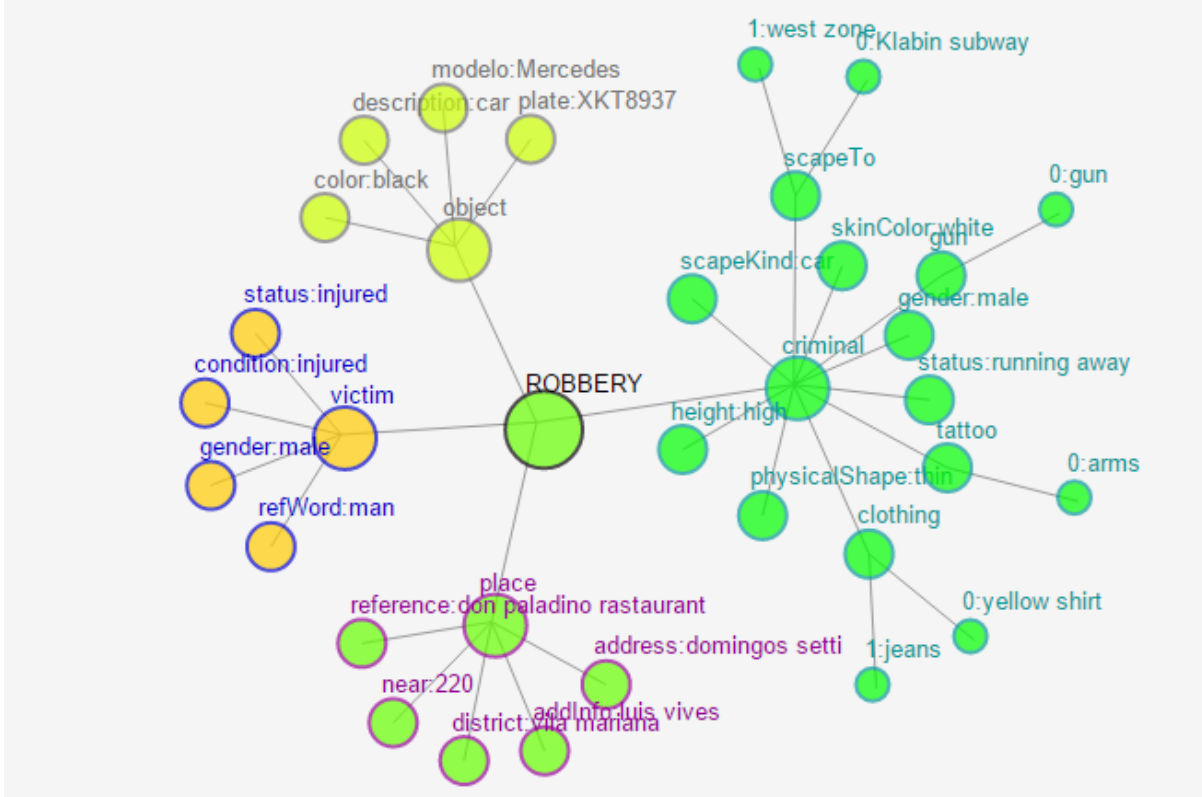

Fig. 4 - Third view, hierarchical graph situational presentation.

\subsection{Presenting Level 2 of Awareness directly}

The goal is to present necessary information into a second SA level that support directly understanding of minimum processing, as a first indication that a situation is probably happening. The idea is to present some figures already calculated instead of relying experts calculation on the Level 1 SA data.

Some situations (composed by objects and attributes) can be calculated and prioritized to reduce operator's mental calculation. For example, the automated part can perform information fusion from multiple objects "object" identified in the acquired information.

Fig. 5 (a) shows a case of robbery, but refers something unidentified. By means of new reports, it is identified that a car was stolen, according to (b), is identified the car's color which is black (c) and the car's brand that is a Mercedes(d). 
So, instead of displaying the input information separately information merged with low dimensionality and more meaning can be adopted.

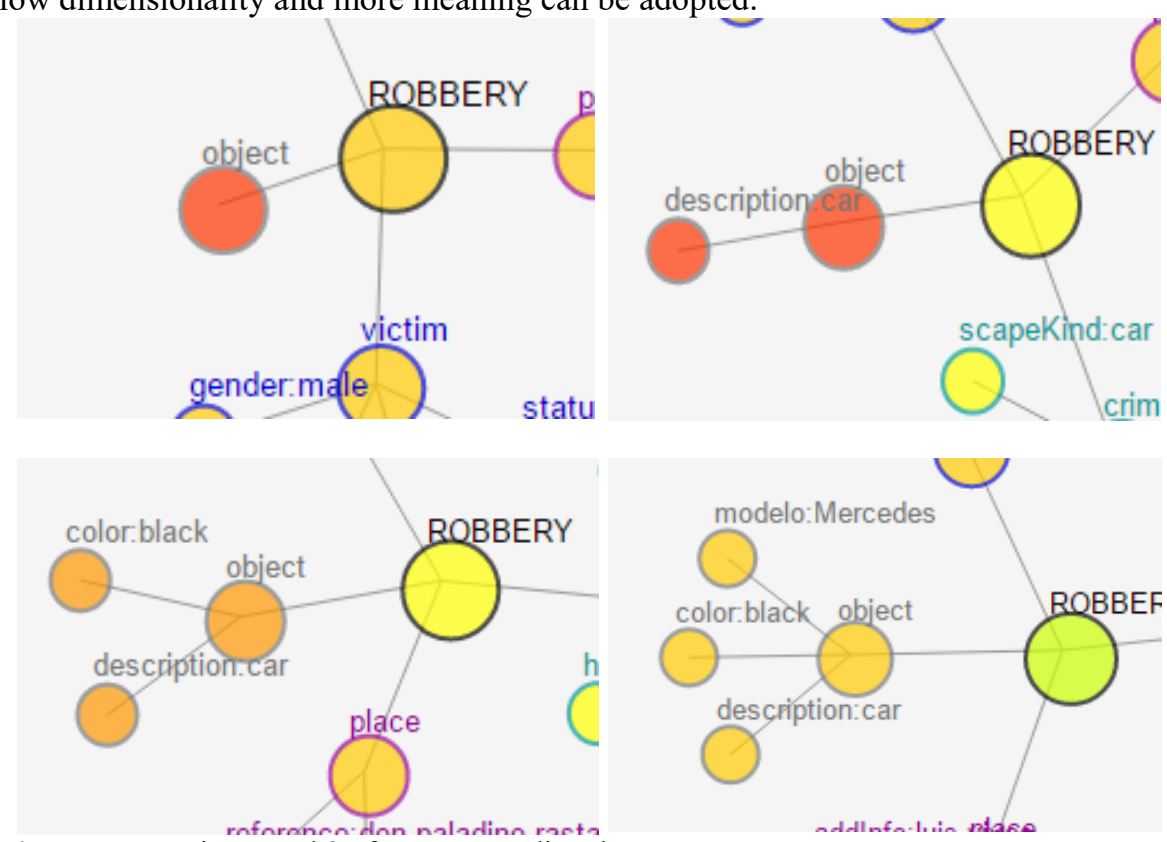

Fig. 5 - Presenting Level 2 of Awareness directly.

\subsection{Supporting Global Situation Awareness}

The "situation picture" should always be available. Global SA is the overview of the whole situation in a high level language and according to experts objectives. It is the data information and information apparently useful to the situation added to apparently not useful information.

In most Situation Assessment systems, global SA is always visible and can be crucial to determine what goals have high priority. In the development scenario of this UI, the overall SA can be represented by the map display, as shown in Fig. 3.

The permanence of these objects and attributes in the situation formation is important because according to the situation and information, the operator can be guided or not in the decision-making process.

\subsection{Information Filtering}

To avoid overloading not SA-related information should be filtered. The UI should only provide crucial information for the purposes of each task. Therefore, an interactive filter was developed.

This filter is useful to reduce search space and determines fusion candidates through visual analysis. However, SA does not occur instantaneously. Humans take time to orient themselves in relation to situations and critical attributes. To this end, 
the object table can be expanded on demand to expose and hide information on the report of a certain insertion of objects, textually, as shown in Fig. 2.

Furthermore, when a relation candidate has been detected, indicated by humans or the system, the table and the graph object provides a new graphical link indicating a probable relationship that may or may not be accepted by the skilled person.

\subsection{Support the Reliability Verification of Information}

It is known that in the context of data usage of human intelligence (HUMINT) as input, there is a great chance that the acquired data present some kind of quality problems.

To infer and represent the data source reliability local quality indices were adopted. The better the quality of the generated information, higher the data source reliability. Although the values reliability can be displayed numerically, Ware [11] states that the use of luminance levels is advised (lighter for more reliable).

Thus, the UI shows alternative forms of representing quality. The lower the information quality, the faster the human operator decision (high, medium, low) and they tend to better accept the lowest rates. The numerical data uses analog and in classification tend to generate slower decisions.

For UI, it is employed the use of colors and shapes, plotting relevant dimensions to the domain and generalization sure of the situation. The closer the node approaches the green color higher the data quality. The closer the red worse the data quality, according to Ware [11] on the perception of color in the representation of information.

\subsection{Explicitly Identify Absence of Information}

Humans deal with the absence of metadata as something positive. If there are positive readings, they find a lost reading is also positive when in fact, they can be extremely conflicting and inaccurate. Human beings act differently when they know if there is a likelihood of something going wrong. The lack of information is usually treated as correct and entrust level.

There are two variations of the problem: the inexistence of danger, which is when the information was analyzed and there is no threat; and when there are no known dangers when there are some places that were not covered or sensory limitations.

In addition, stress and workload can lead people to not pay attention to the lack of information. Some humans are dependent on visual information others only rely on their experience.

In that UI nodes have updated their internal color every time the information quality is corrected, mainly due to completeness, presented as the main information quality problem of information in this area, as Fig. 1.

The central node color in the graph represents certainty in the situation, calculated based on completeness and timeliness dimensions of objects. The color of the nodes of the first level is the quality level of each object, calculated based on completeness and particular object of the present time. The second nodes level of coloration follow the color of the first level, because it is a specialization of the object data.

Visual coloring techniques were used where the red-green scale and its hues qualify information. These are generally used to represent errors and information gaps. In 
this case, used to classify certain index thereof, as shown in the topical support and check the reliability of information.

The node size was used to represent information hierarchy, the highest (status) to the smallest (attributes), as shown in Fig. 5.

In the map, the use of glyphs happens due the abstraction the operator may have about the representation composed by objects in the formation of such display. In this case, a location marker was used to represent the median location in relation to other glyphs situation, as the colors techniques were employed. Glyphs with representation phones and social networks have been used to facilitate understanding of the origin of the information.

The site map was created in white and shades of gray to avoid operator's mental confusion in relation to relief and other information. Their choice is justified by better application grating staining of the glyphs, as shown in Fig. 3.

\subsection{Representing Historical Events to follow up Information Evolution}

In order to move forward and return to the situation evolution the UI displays graphic and interactive access to historical information on a timeline. A timeline was implemented in order to allow users to move along the situation and rediscover how it was at any given time.

In our approach, the situation is something that evolved over time. Past situations can also be restored through access buttons. When triggered, these buttons allow the user to navigate the situation, knowing how it behaved at any given time. The navigation is based on the reports where each number is the entry of a new report.

Thus, there is a possibility of returning in the past and also to monitor events in real time and move directly to a specific time. As a downside, there is a possible loss of focus on relevant current events and confusion about the reality of events.

\subsection{Support quality of data and information level and Uncertainty Management}

As experts need evaluate the utility of situational information, might have a manner for them be able to adapt such information to the necessities of their tasks. In this context, need the human act reflects on the information quality, it is inferred and presents by automation.

Once situational information was previously granted, the attributes referring to information quality are also measured and presented at the UI, together qualified information, in the visual suggestions form (cues) that shows such qualification. In this context, data and information quality not only assists experts to establish a trust level that they need deposit on information, but also guide them to seek resources to improve information quality, complementing the performed automation, but possibly cannot have been sufficient to arouse the human trust and stimulate the SA process.

In this approach, the expert is able to insert and remove parts of situational information, in this way, it is possible that expert insert, update or remove objects and attributes that compose situational awareness directly. The graph allows SA support allowing insertion and removal of news attributes that experts judge relevant for the situation as show in Fig. 6. 


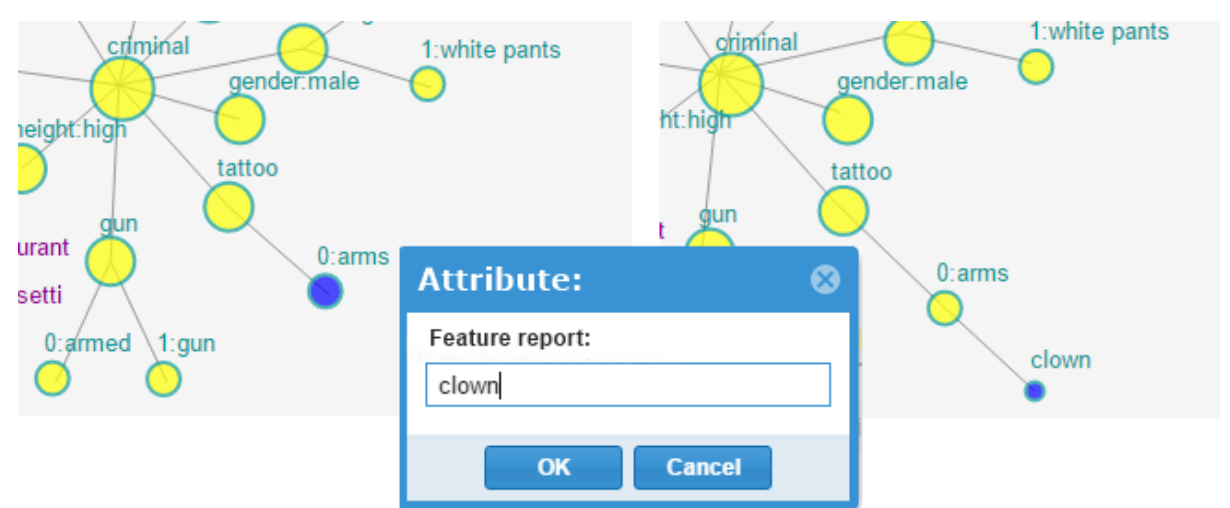

Fig. 6 - Attributes insertion example. The clown attribute been inserted at tattoo attribute. Reference to the existence of a clown tattoo on the criminal arm.

With this option at the tool, the UI expert have the possibility to manipulate the UI generated information, adding a new attribute that judge relevant for the situation or removing an attribute according your necessity.

Acting directly on situational knowledge, entered, updated or removed information reflect in others situational assessment process stages and may be considered for the Assessment, Fusion, Semantic Presentation and Graphical Interface.

\section{Conclusion}

During all Situation Assessment process, the situational information it is advertising, evolves and may be compromised because suffered transformations over time. The information quality in turn may be evaluated throughout the evolution process In this way, the user interface utilization to assists information management control helps the process to acquire, maintain and recover SA.

The UI, is responsible to allow the Situation Knowledge Presentation, where information presentations mechanisms do not interfere information semantically, but allow increment the situation knowledge relation process stages.

Both information has the goal to support SA-oriented UI, which is the situational information management and the contributed process for your formation.

With the information presented, concludes that is possible insert an SA-oriented UI on the emergency situation assessment process, because as shown at case of study the application had satisfactory results, assisting the experts acquire, maintain and recover SA process at UI. This conclusion, is based on the developed assessment and the UI development guidelines.

\section{References}

1. Endsley, Mica R.: Designing for situation awareness: An approach to user-centered design. CRC Press, vol. 56, pp. 727-728. (2011)

2. Kokar, M. M., \& Endsley, M. R. (2012). Situation awareness and cognitive modeling. IEEE Intelligent Systems, (3), 91-96. 
3. Llinas, J., Bowman, C., Rogova, G., Steinberg, A., Waltz, E., \& White, F. (2004). Revisiting the JDL data fusion model II. SPACE AND NAVAL WARFARE SYSTEMS COMMAND SAN DIEGO CA.

4. Baumgartner, N., Gottesheim, W., Mitsch, S., Retschitzegger, W., \& Schwinger, W. (2010). BeAware! - situation awareness, the ontology-driven way. Data \& Knowledge Engineering, 69(11), 1181-1193.

5. Yu, S., Deng, L., \& Zhang, Y. (2009, August). Visualization user interface for decision support systems. In Hybrid Intelligent Systems, 2009. HIS'09. Ninth International Conference on (Vol. 1, pp. 63-66). IEEE.

6. Feng, Y. H., Teng, T. H., \& Tan, A. H. (2009). Modelling situation awareness for Contextaware Decision Support. Expert Systems with Applications, 36(1), 455-463.

7. Souza, J. Botega, L., Santarém Segundo, J.E., Berti, C.: Conceptual Framework to Enrich Situation Awareness of Emergency Dispatchers. In: 17th International Conference on $\mathrm{Hu}-$ man-Computer Interaction, Los Angeles. Lecture Notes in Computer Science (LNCS), (2015)

8. Junior, V., Sanches, M., Botega, L., Souza, J., Saraiva, Caio., Fusco, E., Campos, M., Araújo, R.: Multi-Criteria Fusion of Heterogeneous Information for Improving Situation Awareness on Military Decision Making System. 17th International Conference on HumanComputer Interaction, 2015, Los Angeles. Lecture Notes in Computer Science (LNCS), (2015).

9. M. R. Endsley. "Designing for Situation Awareness: An Approach to User-Centered Design". (pp.19 -21). Boca Raton-FL: CRC Press, 365. 2012.

10.E. Onal, C. Craddock, M. R. Endsley, and A. Chapman, "From theory to practice: How designing for situation awareness can transform confusing, overloaded shovel operator interfaces, reduce costs, and increase safety". ISARC 2013, pp. 1517-1525.

11.Ware, C. (1988). Color sequences for univariate maps: Theory, experiments and principles. Computer Graphics and Applications, IEEE, 8(5), 41-49. 